\title{
Plasma homocysteine levels in patients with Psoriasis
}

\author{
Mousumi Das', Indranil Dawn², Susmita Sarkar³, Kapildev Das ${ }^{4}$ \\ ${ }^{1,2,3}$ Assistant Professor, Department of Biochemistry, ${ }^{4}$ Assistant Professor, Department of Dermatology, Malda Medical \\ College, Malda Town, West Bengal, India
}

Background: Hyper homocysteinaemia represents an independent risk factor for atherosclerotic cardiovascular disease, stroke, peripheral arterial occlusive disease and venous thrombosis. Psoriasis is a chronic inflammatory skin disease associated with increased atherothrombosis and cardiovascular risk profile. Aims and Objective: The aim of this study is to investigate homocysteine in psoriatic patients and its relationship with the severity of the disease. Materials and Methods: A case control study in 50 patients with chronic plaque psoriasis and 50 healthy controls was performed. Total plasma homocysteine level, and PASI index were assessed in every patient. Results: Patients with psoriasis had plasma homocysteine levels higher than controls. The severity of psoriasis assessed according to PASI directly correlate either with plasma homocysteine. Conclusion: A significantly higher prevalence of hyperhomocysteinaemia was found in psoriatic patients compared to healthy controls. Our data directly correlate the high level of homocysteine with higher PASI scores.

Access this article online

Website:

http://nepjol.info/index.php/AJMS

DOI: 10.3126/ajms.v8i5.17136

E-ISSN: 2091-0576

P-ISSN: 2467-9100

Key words: Homocysteine, Psoriasis, PASI scores

\section{INTRODUCTION}

Psoriasis is a chronic inflammatory skin disease associated with increased atherothrombosis and cardiovascular risk profile. It is a hyperproliferative, cutaneous condition with the potential to lower levels of folate. This may result in hyperhomocysteinaemia that represents an independent risk factor for atherosclerotic cardiovascular disease, stroke, peripheral arterial occlusive disease and venous thrombosis.

Psoriasis is a chronic and recurrent inflammatory skin disease characterized by erythematous scaling plaques, affecting $1-5 \%$ of the general population. ${ }^{1}$ An association between psoriasis and cardiovascular morbidity and mortality has been reported. ${ }^{2}$ Furthermore, cardiovascular risk factors, such as dyslipidaemia, ${ }^{3}$ hypertension ${ }^{4}$ oxidative stress $^{5}$ diabetes mellitus ${ }^{6}$ and metabolic syndrome ${ }^{7}$ are more prevalent among patients with psoriasis, increase their cardiovascular risk profile. Homocysteine is a sulphurcontaining amino acid generated by the catabolism of methionine. It is largely catabolized by trans-sulphuration to cysteine but it may also be re-methylated to methionine.
Vitamin B12, vitamin B6 and folate are important cofactors in its metabolism. ${ }^{8}$ Plasma homocysteine is now established as a clinical risk factor for coronary artery disease, as well as other arterial and venous occlusive diseases. Homocysteine is thought to have thrombophilic properties due to an oxidative stress damaging vascular endothelium. ${ }^{9}$ For this reason, hyperhomocysteinaemia may constitute an independent risk factor for cardiovascular disease. ${ }^{10-11}$ Since many studies have demonstrated the presence of an increased homocysteinaemia value in psoriatic patients and considering that psoriasis is a disease associated with increased atherothrombosis, the aim of this study is to investigate the relationship between hyperhomocysteinaemia and psoriasis. The association between homocysteine and the severity of psoriasis was also evaluated.

\section{MATERIALS AND METHODS}

This case control study was conducted in Malda Medical College and Hospital, a rural based medical college and 
hospital in West Bengal (India). Forty patients as cases were selected for the study.

\section{Inclusion criteria}

The patients were selected with history of red and itchy plaques over the skin covered with silver colored scales. The skin lesions are remitting and relapsing in nature.

\section{Exclusion criteria}

Subjects with any chronic illness, cancer, hyperuricemia were not included in the study. Patients taking any medications like corticosteroids, methotrexate, and with history of smoking and alcoholism were also excluded from the study.

Disease's severity and coverage were assessed according to the Psoriasis Area and Severity Index (PASI). PASI score was applied to assess the severity and coverage of chronic plaque psoriasis, and the determinations were carried out at the time of the first evaluation of the patients. The PASI assesses the redness, thickness and scale of the psoriasis lesions, on a scale from 0 (none) to 4 (severe) for each of these attributes. The percentage of skin covered by psoriasis is also scored on a scale from $0(0 \%)$ to $6(90-100 \%)$. This is done for each of the trunk; head, arms and legs, and the scores are weighted by the location of psoriasis.

The procedures followed were in accordance with the ethical standards of the responsible committee on human experimentation (institutional) and with the Helsinki Declaration of 1975, as revised in 1983.

\section{Principle of serum homocysteine assay}

Homocysteine assay is based on the measurement of cosubstrate conversion product. Oxidized homocysteine from the sample is reduced to free homocysteine which then reacts with SAM to form methionine and SAH. SAH is assessed by coupled enzyme reactions wherein adenosine is formed. The adenosine formed is hydrolysed into inosine and ammonia which reacts with glutamate dehydrogenase with concomitant conversions of NADH to NAD + . The concentration of homocysteine is proportional to the amount of $\mathrm{NADH}$ converted to NAD + and is measured as change in absorbance at $340 \mathrm{~nm}$.

\section{RESULT}

Plasma homocysteine levels were significantly higher in patients with psoriasis than in controls (mean $=12.43$ vs 7.38 umol $/ \mathrm{L} ; \mathrm{SD}=1.9$ vs. $0.6 ; \mathrm{t}=-17.9 ; \mathrm{P}=<0.001$ ) (Table 1 , Figures 1 and 2).

Considering the correlation between hyperhomocisteinaemia and psoriasis severity assessed by PASI, it directly

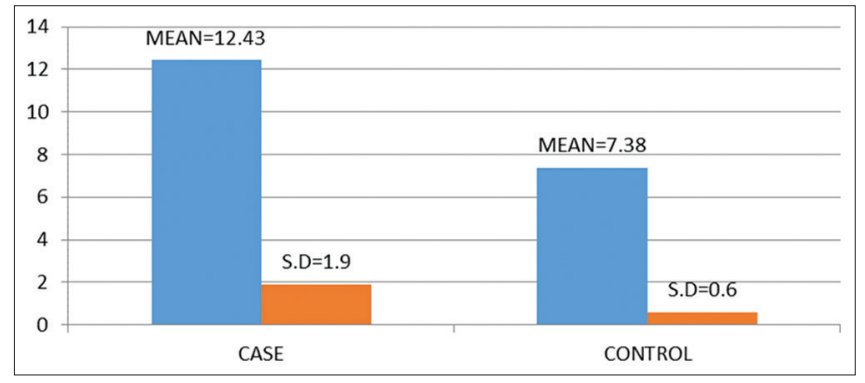

Figure 1: Plasmatic value of homocysteine of case and control (Bar diagram)

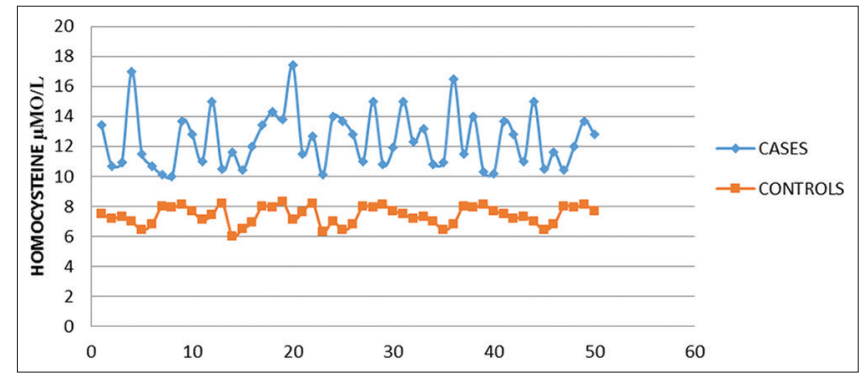

Figure 2: Plasmatic value of homocysteine of case and control (Scatter diagram)

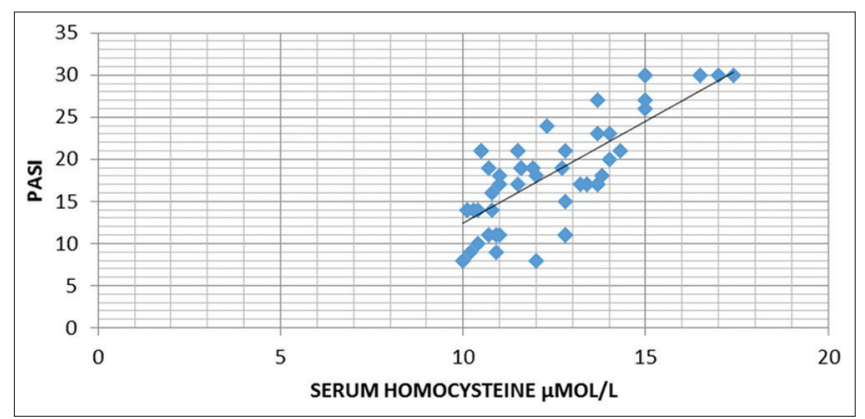

Figure 3: Correlation between homocysteine value (cases) and pasi score

\begin{tabular}{|c|c|c|c|}
\hline Subjects & Mean \pm SD & $P$ value & $t$ value \\
\hline $\begin{array}{l}\text { Case } \\
\text { Control }\end{array}$ & $\begin{array}{c}12.43 \pm 1.9 \\
7.38 \pm 0.6\end{array}$ & $<0.001$ & 17.9 \\
\hline
\end{tabular}

SD: Standard deviation

\begin{tabular}{|c|c|c|}
\hline Subjects & $\begin{array}{l}\text { Correlation } \\
\text { coefficient }\end{array}$ & $P$ value \\
\hline $\begin{array}{l}\text { Correlation between homocysteine } \\
\text { value (cases) and PASI SCORE }\end{array}$ & 0.748 & $<0.01$ \\
\hline
\end{tabular}

correlate with homocysteine $(\mathrm{p}$ value $=<0.01$, Correlation coefficient $=0.748)($ Table 2 and Figure 3$)$. 


\section{DISCUSSION}

Psoriasis is a common chronic and recurrent inflammatory skin disorder that has been associated with abnormal plasma lipid metabolism ${ }^{12}$ high frequency of cardiovascular events ${ }^{13}$ and alterations in the risk of thromboembolic events ${ }^{14}$. In the literature, there are several studies that investigated homocysteine plasma levels in psoriatic patients. Refsum et al. in 1989 reported a higher homocysteine plasma level and lower folic acid level in 13 psoriatic patients ${ }^{15}$. Kural et al. studied 30 psoriatic patients and 30 controls; they found higher homocysteine plasma levels. Malerba et al. reported higher homocysteine in 40 psoriatic patients compared to 30 controls.

Our study, carried out on 50 psoriatic patients and 50 controls, showed significantly high plasma homocysteine levels among the psoriatic group (Table 1, Figures 1 and 2). Data from the literature confirm our results; several studies have shown that the homocysteine plasmatic concentration in psoriatic patients is higher than in healthy controls however, the correlation we found between homocysteine serum levels and psoriasis severity measured by PASI score has hardly ever been reported in the literature. Few studies show a relationship between homocysteine blood levels and disease activity. Physicians must find a pharmacological treatment that reduces the plasmatic homocysteine levels, even when these are only moderately high; we know that folic acid, vitamin B6 and vitamin B12 are all involved in breaking down homocysteine in the blood. Therefore dietary supplementation with folic acid and vitamins B6 and B12 is recommended. Treatment of hyperhomocysteinaemia may not only reduce atherosclerotic plaque areas, but also it may decrease the risk of ischemic heart disease, deep vein thrombosis and stroke. Based on their findings, the authors suggest that psoriatic patients should be treated by a global management taking into account cardiovascular risk factors.

Considering the correlation between hyperhomocysteinaemia and psoriasis severity assessed by PASI, it directly correlate with homocysteine. (Table 2 and Figure 3) Malerba et al. and Cakmak et al. found a direct correlation between PASI severity and high levels of homocysteine.

\section{CONCLUSION}

Psoriatic patients showed significantly high plasma homocysteine and significant correlation with psoriasis severity assessed by PASI. Physicians must find a pharmacological treatment that reduces the plasmatic homocysteine levels, even when these are only moderately high treatment of hyperhomocysteinaemia may not only reduce atherosclerotic plaque areas, but also it may decrease the risk of ischemic heart disease, deep vein thrombosis and stroke. ${ }^{16}$ Based on their findings, the authors suggest that psoriatic patients should be treated by a global management taking into account cardiovascular risk factors.

\section{Abbreviations}

SAM- S adenosylmethionine

SAH- S adenosylhomocysteine

\section{REFERENCES}

1. Lebwohl M. Psoriasis. Lancet 2003; 361: 1197-1204.

2. Cakmak SK, Gill O, Kilir C, Gonul M, Soylu S and Kilic A. Homocysteine, vitamin B12 and folic acid levels in psoriasis patients. J Eur Acad Dermatol Venereol 2009; 23:300-303.

3. Brenner S, Krakowski A, Levtov O, Heldenberg D, Werbin B and Tamir I. Serum lipids in patients with psoriasis. Dermatologica 1975; 150(2):96-102.

4. Lindegard B. Diseases associated with psoriasis in a general population of 159200 middle aged, urban, native Swedes. Dermatologica 1986; 172(6):298304.

5. Trouba KJ, Hamadeh HK, Amin RP and Germolec DR. Oxidative stress and its role in skin disease. Antioxid Redox Signal 2002; 4(4):665-673.

6. Binazzi M, Calandra P and Lisi P. Statistical association between psoriasis and diabetes: further results. Arch Dermatol Res 1975; 254(1):43-48.

7. Gisondi P, Tessari G, Conti A, Piaserico S, Schianchi S and Peserico A. Prevalence of metabolic syndrome in patients with psoriasis: a hospital-based case-control study. Br J Dermatol 2007; 157(1):68-73.

8. Wierzbicki AS. Homocysteine and cardiovascular disease: a review of the evidence. Diab Vase Dis Res 2007; 4(2): 143-150.

9. Refsum H, Ueland PM, Nygard $\mathrm{O}$ and Vollset SE. Homocysteine and cardiovascular disease. Annu Rev Med 1998; 49:31-62.

10. Lonn E, Yusuf S, Arnold MJ, Sheridan P, Pogue J and Micks M. Homocysteine lowering with folic acid and vitamins in vascular disease. New Engl J Med 2006; 354(15):1567-1577.

11. Langman LJ and Cole DE. Homocysteine: cholesterol of the 90s? Clin Chim Acta 1999; 286(1-2):63-80.

12. Rocha-Pereira $P$, Santos-Silva A, Rebelo I, Figueiredo A, Quintanilha A and Teixeira F. Dislipidemia and oxidative stress in mild and in severe psoriasis as a risk for cardiovascular disease. Clin Chim Acta 2001; 303(1-2):33-39.

13. Wakkee M, Thio HB, Prens EP, Sijbrands EJ and Neumann HA. Unfavorable cardiovascular risk profiles in untreated and treated psoriasis patients. Atherosclerosis 2007; 190(1): 1-9.

14. Marongiu F, Sorano GG, Bibbo C, Pistis MP, Conti M, Mulas P, et al. Abnormalities of blood coagulation and fibrinolysis in psoriasis. Dermatology 1994; 189(1):32-37.

15. Refsum $\mathrm{H}$, Helland $\mathrm{S}$ and Ueland $\mathrm{PM}$. Fasting plasma homocysteine as a sensitive parameter of antifolate effect: a study of psoriasis patients receiving low dose methotrex. Clin Pharmcaol Ther 1989; 46(5): 510-520.

16. Malerba M, Gisondi $P$, Radaeli $A$, Sala R, Calzavara Pinton PG and Girolomoni G. Plasma homocystiene and folate levels in patients with chronic plaque psoriasis. Br J Dermatol. 2006; 155:1165-1169. 
Authors Contribution:

M.D - Concept and design of the study, manuscript preparation, statistically analyzed and interpreted, critical revision of the manuscript; I.D - Concept and design of the study, critical revision of manuscript and review of the study; S.S - reviewed the literature, helped in preparing first draft of manuscript, collected data; K.D - collected data, statistically analyzed and interpreted, helped in preparing first draft of manuscript.

\section{Orcid ID:}

Dr. Mousumi Das: http://orcid.org/0000-0002-6698-110X

Dr. Indranil Dawn:(1) http://orcid.org/0000-0002-4152-8421

Dr. Susmita Sarkar:D http://orcid.org/0000-0002-9352-7586

Dr. Kapildev Das: (iD http://orcid.org/0000-0001-8358-2488 